\title{
Mechanically stable kirigami deformable resonant circuits for wireless vibration and pressure sensor applications
}

Srinivas Gandla, ${ }^{1}$ Jaewoo Song, ${ }^{1}$ Jonghwan Shin, ${ }^{1}$ Seungho Baek, ${ }^{1}$ Minwoo Lee, ${ }^{1}$ Danial Khan, ${ }^{2}$ Kang-Yoon Lee, ${ }^{2}$ Jung Ho Kim, ${ }^{3}$ and Sunkook Kim ${ }^{1 *}$

${ }^{1}$ Multifunctional Nano Bio Electronics Lab, Department of Advanced Materials Science and Engineering, Sungkyunkwan University, Suwon 16419, South Korea.

${ }^{2}$ Department of Electrical and Computer Engineering, Sungkyunkwan University College of Information and Communication Engineering, Suwon 16419, South Korea.

${ }^{3}$ Institute for Superconducting and Electronic Materials, Australian Institute for Innovative Materials, University of Wollongong, Squires Way, North Wollongong, NSW, 2500 Australia.

\section{Corresponding Author}

Email: seonkuk@skku.edu 


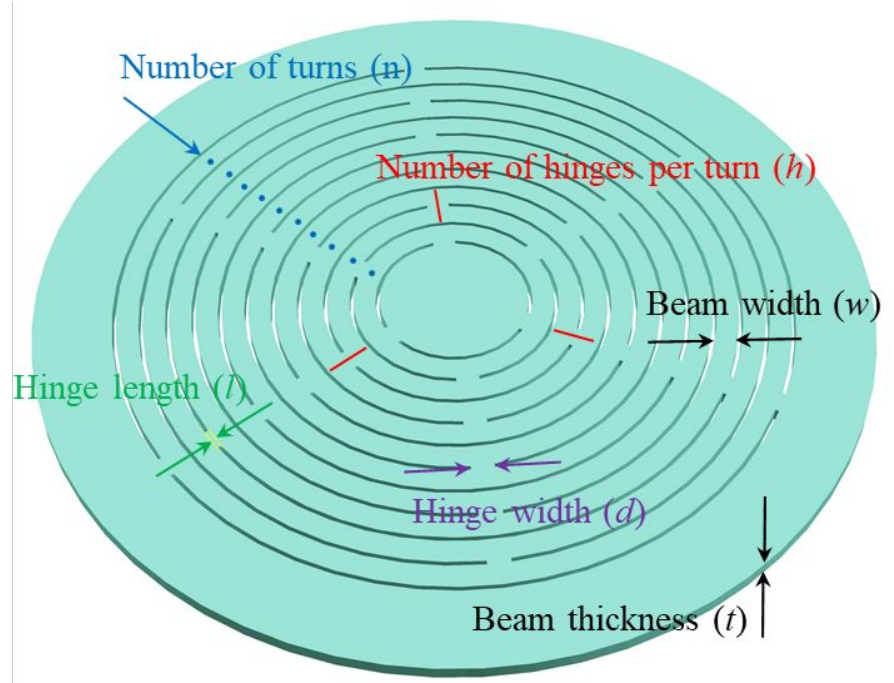

Figure S1. Structural parameters of mechanically deformable circular kirigami: beam width (w), beam thickness (t), number of turns (n), hinge length (1), hinge width (d), and number of hinges per turn (h). 


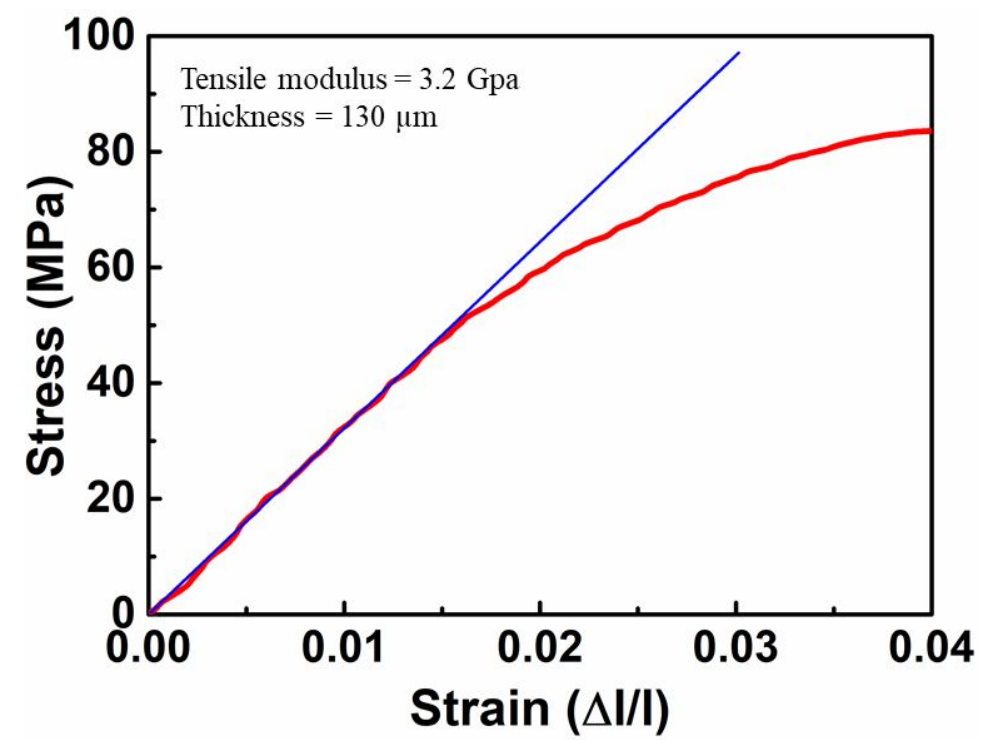

Figure S2. Stress versus strain plot to extract the tensile modulus of $130 \mu \mathrm{m}$-thickness PET film used to fabricate the sensor. 


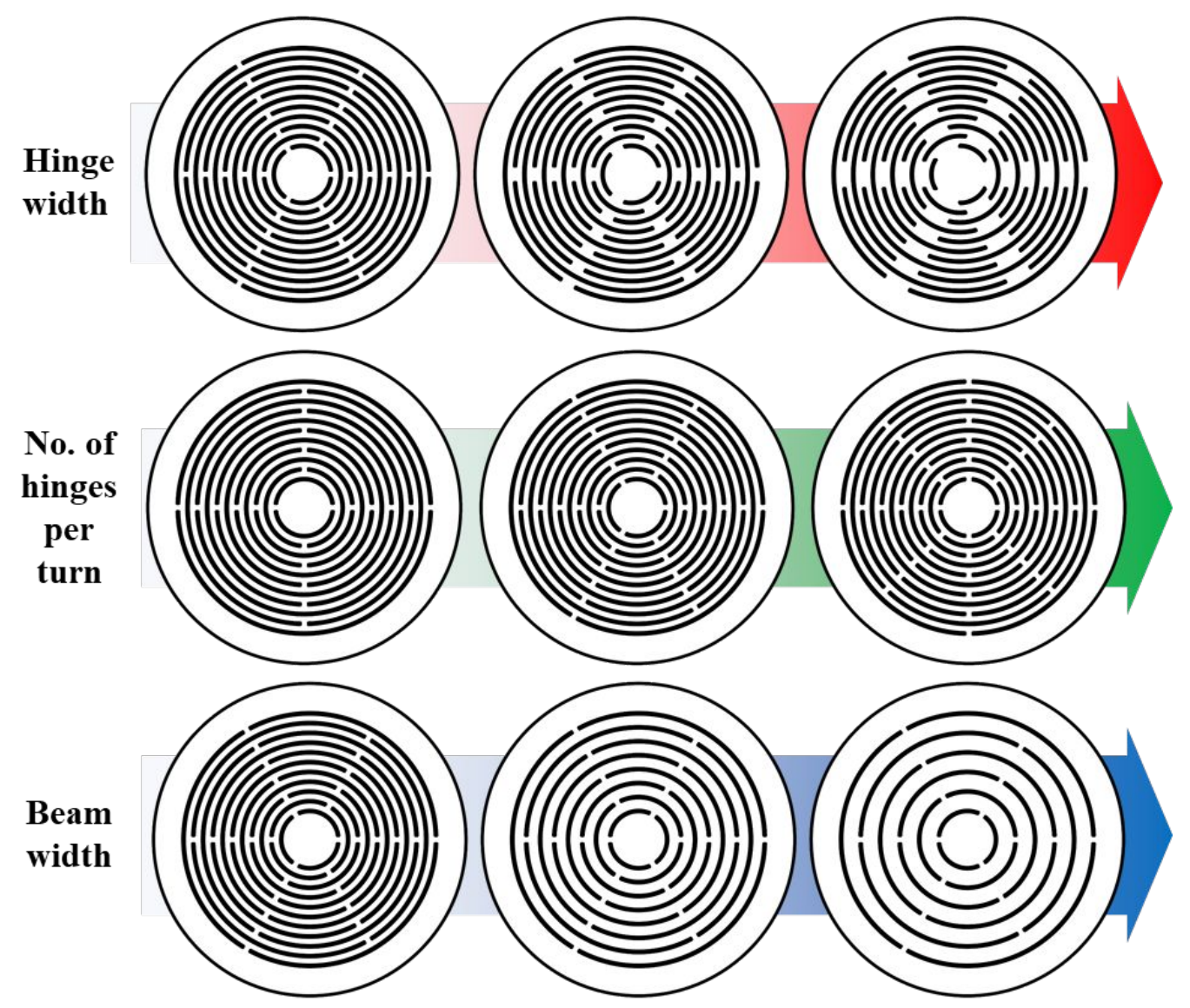

Figure S3. Tunable mechanical stiffness of mechanically deformable circular kirigami structures by separately varying hinge width (d), number of hinges per turn $(\mathrm{h})$, and beam width $(\mathrm{w})$. 


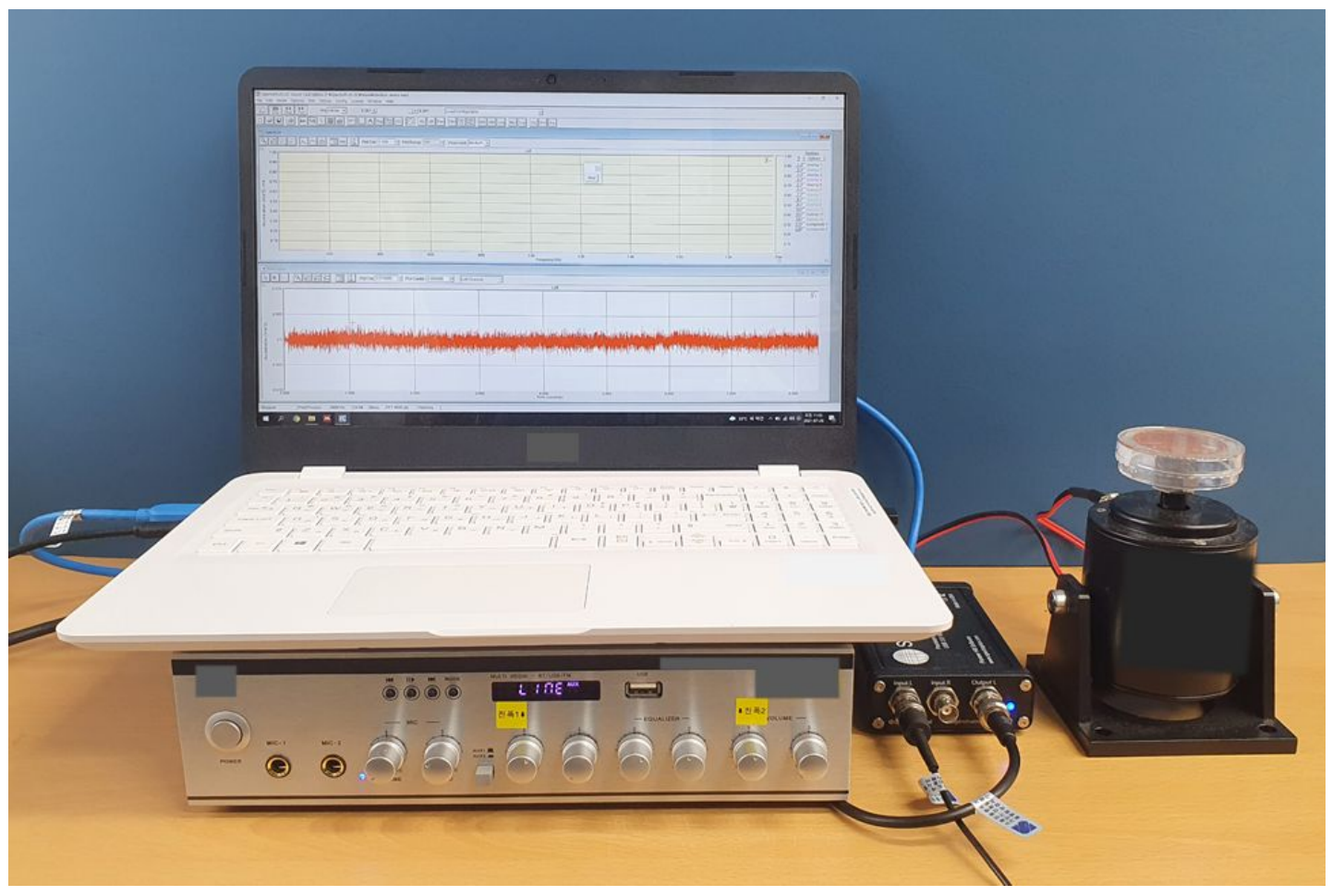

Figure S4. Vibration monitoring setup using YMC Modal Shaker combined with function generator and amplifier. 

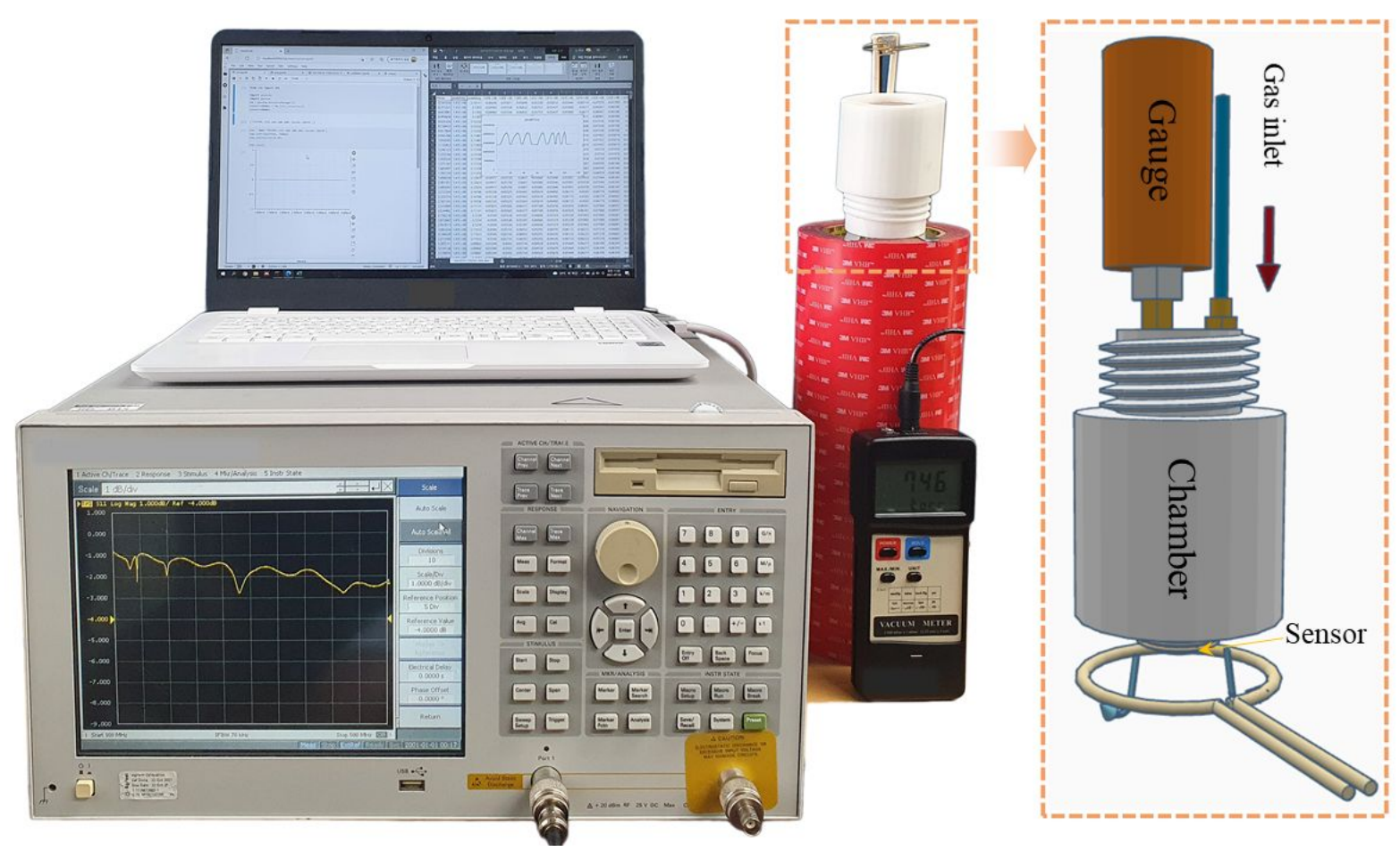

Figure S5. Real-time monitoring of wireless resonant frequency response of the pressure sensor to the applied pressures measured using a Python-program-controlled vector network analyzer. 